\title{
Criminologie
}

\section{La Cité des Prairies : 25 ans de réalisations}

Équipe de Cité des Prairies

Volume 21, numéro 1, 1988

Entre les mailles de la loi : pollution, accident de travail, faute professionnelle

URI : https://id.erudit.org/iderudit/017261ar

DOI : https://doi.org/10.7202/017261ar

Aller au sommaire du numéro

Éditeur(s)

Les Presses de l'Université de Montréal

ISSN

0316-0041 (imprimé)

1492-1367 (numérique)

Découvrir la revue

Citer cet article

Équipe de Cité des Prairies (1988). La Cité des Prairies : 25 ans de réalisations.

Criminologie, 21(1), 103-119. https://doi.org/10.7202/017261ar d'utilisation que vous pouvez consulter en ligne.

https://apropos.erudit.org/fr/usagers/politique-dutilisation/ 
Dans les marges de l'actualité

\section{LA CITÉ DES PRAIRIES: 25 ANS DE RÉALISATIONS} Équipe de Cité des Prairies*

La Cité des Prairies qui fête cette année son $25^{\mathrm{e}}$ anniversaire est un centre d'accueil de réadaptation qui reçoit, en internat et en externat, près de 270 adolescents présentant de graves problèmes de comportement ou de mésadaptation sociale. Certains font l'objet d'une mesure de détention provisoire, de garde fermée ou de garde ouverte dans le cadre de la Loi sur les jeunes contrevenants; d'autres font l'objet d'une intervention en vertu de la Loi sur la protection de la jeunesse. Voilà pour la présentation officielle...

Pour les partenaires du réseau, Cité des Prairies c'est «le bout de ligne», la dernière tentative de faire quelque chose pour ces adolescents de 15 à 18 ans, dont une majorité a déjà complété le circuit des familles d'accueil et des centres de réadaptation.

Ce sont des jeunes dont on dit qu'ils «représentent un haut degré de dangerosité pour la société ou pour eux-mêmes». Plusieurs ont à leur passif de multiples délits et sont déjà profondément engagés dans une délinquance qui n'est pas toujours exempte de violence. Certains connaissent surtout des problèmes graves de comportement qui se manifestent souvent par des retards scolaires importants. Un bon nombre d'entre eux sont des fugueurs invétérés pour lesquels l'intervention en milieu ouvert s'est avérée inefficace.

Les adolescents qui sont admis à Cité des Prairies y arrivent donc après plusieurs années de difficultés personnelles et de confrontations majeures avec leur famille, leur entourage et les institutions - scolaires, judiciaires, juridiques, sociales - qu'ils ont connues jusque-là. Quand on dit qu'ils «échouent» à Cité des Prairies, après souvent plusieurs récidives et quatre ou cinq placements antérieurs, on cerne peut-être la réalité de plus près qu'on le croit. Ces adolescents vivent effectivement l'arrivée à Cité des Prairies comme l'aboutissement d'une série d'échecs. Ils sont révoltés, bien sûr, mais ils sont surtout brisés. Ce qui les caractérise par-dessus tout, c'est une perte totale de confiance: dans les adultes, évidemment, - particulièrement ceux, nombreux, qui sont

* Centre d'accueil la Cité des Prairies, 12 165, boulevard Saint-Jean-Baptiste, Montréal, Québec HIC 1S4.

Criminologie, XXI, 1 
«intervenus» auprès d'eux - mais surtout perte de confiance en euxmêmes, en leur capacité de s'en sortir.

Devant un tel esprit, où se mélangent révolte, méfiance, désespoir, cynisme et résignation, la Cité des Prairies devra faire beaucoup plus que d'assurer la protection de la société en contrôlant l'agir délinquant et en mettant fin aux comportements antisociaux.

Mettre l'adolescent face à ses responsabilités sociales, bien sûr, mais surtout lui redonner confiance dans cette société en lui prouvant qu'il a en lui le potentiel nécessaire pour s'y tailler une nouvelle place, voilà comment Cité des Prairies interprète son mandat.

\section{L'ACCUEIL}

Personne n'entreprend de gaieté de cour un séjour en institution. Même si ce séjour repose sur un choix ou un consentement, la personne âgée ou le malade chronique qui arrive dans son nouveau milieu de vie a besoin d'y être préparé et d'y être accompagné.

Sa situation présente certaines similitudes avec celle de l'adolescent qui est admis à Cité des Prairies, bien que l'âge de ce dernier, l'état de crise et de révolte où il se trouve, l'absence complète de liberté dans sa décision et l'encadrement contraignant dont il fait l'objet accroissent de beaucoup ses difficultés d'adaptation.

Face à cette phase difficile d'adaptation et d'acceptation par l'adolescent de son nouveau milieu de vie, Cité des Prairies a choisi de jouer cartes sur table en informant le jeune de tous les aspects de son séjour et en concluant avec lui une entente claire sur ce qu'il est en droit de s'attendre des éducateurs et du Centre, et, en contrepartie, de ce que le Centre exigera de lui.

Chaque adolescent dont le dossier est acheminé à Cité des Prairies pour admission est donc rencontré personnellement dans le milieu où il séjourne. Lors de cette rencontre - ou de ces rencontres, selon les besoins exprimés par l'adolescent - les «règles du jeu» sont explicitées et débattues. Le jeune est invité à une visite préliminaire du Centre, visite à laquelle il peut également convier ses parents.

Bien sûr, ces rencontres n'ont pas pour seul but d'informer l'adolescent. Elles visent surtout à démystifier le Centre que plusieurs jeunes perçoivent souvent comme «le maximum» du réseau, confondant allègrement ce qu'ils ont entendu raconter sur les pénitenciers avec la réadaptation en milieu fermé. 
Quand ils apprennent, par exemple, qu'ils disposeront d'une chambre privée - sur la décoration de laquelle ils auront leur mot à dire - qu'ils pourront porter leur propres vêtements et avoir éventuellement accès à leur «système de son», qu'ils pourront recevoir deux fois la semaine des visites de leur famille, plusieurs des préjugés les plus tenaces s'estompent.

Il serait exagéré de prétendre que les jeunes arrivent à Cité des Prairies en sifflotant. Mais l'effort mis sur la préparation de leur séjour a sans contredit permis de gagner un temps précieux lors de la période de latence et de retrait qui marque toujours l'intégration à une institution. Avec l'évolution judiciaire récente, qui tend à une réduction de la durée des ordonnances, ces quelques semaines gagnées en début de processus de réadaptation s'avèrent d'autant plus précieuses qu'elles permettront souvent d'enclencher plus rapidement la phase décisive de la réinsertion sociale, véritable but de toute l'intervention.

\section{LA RÉADAPTATION : UN CHOIX}

Lors des rencontres préliminaires à l'admission, et pendant les premières semaines du séjour de l'adolescent, les éducateurs en contact avec le jeune lui véhiculeront un message clair et cohérent: $s$ 'il est vrai que l'adolescent n'a pas choisi de vivre à Cité des Prairies, c'est finalement sur lui que repose la décision de saisir l'opportunité que peuvent représenter ces quelques mois au Centre. Il peut choisir de «faire du temps» ou il peut se prendre en main et rattrapper une partie de son retard scolaire, apprendre un métier, travailler sur des difficultés personnelles qu'il traîne depuis des années.

C'est souvent lorsqu'il découvre l'étendue et la variété des programmes et, surtout, la possibilité qu'il a de faire valoir son point de vue sur l'orientation qu'il veut donner à son séjour, que l'adolescent «embarque».

Au fond, toute la philosophie d'accueil de Cité des Prairies repose sur ce choix de l'adolescent. Bien évidemment, l'ordonnance du Tribunal est incontournable; le mandat de protection de la société et l'encadrement sécuritaire ne peuvent faire l'objet de négociations; les efforts des éducateurs pour responsabiliser l'adolescent, lui faire prendre conscience de ses difficultés et l'inciter à travailler sur ses problèmes de comportement ne se relâcheront pas. Pourtant, malgré ce contexte, l'adolescent peut choisir d'embarquer ou pas. C'est par un ensemble de programmes et d'interventions personnalisées, dont il sera question dans la partie deux, qu'on l'incitera à le faire. 


\section{LA PLACE DE LA FAMILLE}

L'adolescent qui arrive à Cité des Prairies se trouve généralement dans une situation délicate face à sa famille. Au pire, ces relations sont complètement coupées ou carrëment antagonistes. Plus fréquemment, elles sont marquées par la difficulté de communication ou la culpabilité des deux parties.

Cité des Prairies croit qu'une réadaptation et une réinsertion sociale véritables ne sont possibles que lorsque le jeune a "clarifié» cette situation familiale. Cela ne signifie pas nécessairement un retour au foyer ou même une réconciliation mais, à tout le moins, une compréhension claire des deux parties sur la nature des difficultés.

La participation des parents, tant au plan d'intervention du jeune qu'à l'orientation générale des programmes, se fera donc dans cet esprit: la reprise de la communication, la discussion franche des problèmes, la clarification des attentes mutuelles.

Cette participation des parents débutera, comme nous l'avons déjà mentionné, dès avant l'admission du jeune. Les parents qui le désirent pourront alors visiter le Centre et rencontrer un travailleur social qui leur expliquera le fonctionnement de l'institution et ses objectifs. Cette simple visite est parfois décisive puisqu'elle permet souvent de vaincre les réticences normales que peuvent avoir des parents dont le fils vient d'être «placé en centre fermé».

\section{LES «MULTIS»}

Ce premier contact avec les parents n'est qu'une étape préliminaire à un engagement beaucoup plus significatif et déterminant: leur participation aux «multis».

Les comités multidisciplinaires, que les jeunes préfèrent appeler «multis», sont la pierre d'angle de tout le processus clinique d'intervention.

Organisé un mois après l'arrivée du jeune au Centre, - et, par la suite, à des intervalles de six mois - le comité multidisciplinaire est le mécanisme privilégié pour définir les objectifs du plan d'intervention auprès de l'adolescent. Réunissant le jeune, ses parents et tous les intervenants alors en contact avec lui (éducateur-parrain, travailleur social, professeur, psychologue), le «multi» a pour but de faire le point sur la situation de l'adolescent (pourquoi est-il au Centre? comment lui et ses parents voient-ils ses difficultés? etc.) et sur les objectifs qu'il voudrait atteindre pendant les prochains six mois. 
Cette rencontre, qui peut parfois durer plusieurs heures, ne doit pas être confondue avec une «thérapie familiale». Pourtant, elle n'en est pas moins l'occasion pour le jeune et ses parents, souvent pour la première fois, d'enclencher une discussion et de tirer au clair des problèmes parfois vieux de plusieurs années.

La participation des parents à ces «multis» est évidemment essentielle. Elle leur permet non seulement de mieux connaître les difficultés de leur fils mais aussi de comprendre le travail des éducateurs, de le soutenir et, surtout, d'avoir un mot décisif à dire sur son orientation.

Tout au long du processus de réadaptation, mais particulièrement quand seront abordés les objectifs de réinsertion sociale de l'adolescent (sorties, séjours provisoires dans la famille, retour ou non au foyer), cette présence des parents aura un impact déterminant.

\section{LE COMTTÉ DE PARENTS}

La participation des parents ne se fait pas sur cette seule base individuelle, aussi essentielle soit-elle. Depuis plusieurs années déjà les parents sont appelés à participer collectivement aux orientations mêmes du Centre par l'entremise d'un Comité de parents, qui regroupe des dizaines de parents tous les mois.

Piloté par un exécutif, ce comité est régulièrement consulté sur les réformes apportées aux règlements de l'établissement ou sur l'introduction de nouveaux programmes. Récemment, par exemple, les parents ont ainsi influencé de façon décisive les nouvelles politiques relatives aux visites, aux sorties, et à la «rémunération» des adolescents.

Le comité de parents ne se cantonne évidemment pas dans un rôle passif, attendant d'être consulté pour dire son mot. Pour ne citer qu'un exemple, ce sont les parents qui ont contribué à sensibiliser la direction du Centre au développement des services externes. Premiers témoins des difficultés rencontrées par leur adolescent à sa sortie du Centre, ce sont les parents qui ont amené Cité des Prairies à s'engager directement dans la formation professionnelle, la mise sur pied d'ateliers de travail et l'élaboration de programmes de soutien à l'externat. Il n'est pas exagéré de dire que sans cette pression du Comité des parents, «l'ouverture» du Centre sur la réinsertion n'aurait pas connu les progrès spectaculaires qui l'a caractérisée depuis quelques années.

Par leur engagement au côté de leur fils dans le plan d'intervention, leur participation à des activités spéciales, leur intérêt dans l'éla- 
boration et la mise en place des programmes du Centre, leur travail de mobilisation et de soutien auprès de leurs pairs, les parents de Cité des Prairies font maintenant partie intégrante du Centre et verraient mal qu'on ne les considère pas, au sens strict, comme de véritables intervenants dans le processus de réadaptation et de réinsertion sociale de leurs adolescents.

\section{L'APPORT DES BÉNÉVOLES ET DE LA COMMUNAUTÉ}

L'ouverture vers l'extérieur dont a fait preuve Cité des Prairies depuis quelques années ne s'est pas encore traduite par une présence quotidienne de bénévoles ou de groupes de la communauté auprès des jeunes. Cela peut sans doute s'expliquer par le mandat très précis d'encadrement de l'établissement et les difficultés éminemment concrètes que représente la présence «d'étrangers» à l'intérieur d'un "centre sécuritaire». Il n'en reste pas moins que des efforts particuliers devront être faits à ce niveau. Certains projets à l'étude dans le domaine des loisirs viendront d'ailleurs probablement corriger ces lacunes d'ici peu.

Mais l'absence de bénévoles extérieurs «sur le plancher», ne signifie pas que le bénévolat est absent de Cité des Prairies. Ce serait faire fi du travail remarquable accompli depuis plusieurs années par la Fondation de Cité des Prairies. Si ce sont les parents qui ont «poussé dans le dos» du Centre pour qu'il intervienne à l'extérieur, c'est souvent la Fondation qui lui a fourni les moyens de le faire.

Ce sont en effet les membres de cette Fondation qui ont trouvé les fonds et l'expertise nécessaires pour lancer des initiatives comme la vente de bois de chauffage, l'endossement de jeunes dans l'entreprise privée, la mise sur pied du «Regain», foyer d'accueil pour des expensionnaires de Cité qui rencontrent des difficultés passagères. Toute cette expertise consacrée à la levée de fonds et à la gestion de projets est la forme qu'a empruntée jusqu'à maintenant le bénévolat à Cité des Prairies. Si cette action ne se fait pas directement auprès des jeunes, cela ne les empêche pas d'en être pourtant les bénéficiaires immédiats.

\section{INDIVIDUALISATION, AUTONOMIE ET RESPONSABI- LISATION}

On ne saurait apprécier les mesures d'individualisation mises en place à Cité des Prairies sans les resituer dans la structure clinique générale, dont elles ne sont qu'un aspect. À Cité des Prairies, l'atten- 
tion accordée au respect de l'individu dépasse le niveau des attitudes: elle est, par définition, l'un des principes fondamentaux du processus de réadaptation.

Cité des Prairies a cessé d'être une «prison pour jeunes» ou un «centre sécuritaire» le jour où on a mis en place une structure clinique reposant sur la démarche personnelle de l'adolescent. Depuis ce temps, ce modèle d'intervention, qui table sur les capacités de chaque jeune, n'a cessé de se raffiner. C'est en ce sens que nous disons que les mesures d'individualisation ne peuvent être vues comme «ce petit quelque chose en plus», cette «petite touche» qui viendrait humaniser l'intervention : elles sont au cœur même de cette intervention.

Les deux principes à la base de l'approche clinique de Cité des Prairies sont la responsabilisation et le développement de l'autonomie. Par responsabilisation, nous entendons l'effort de l'adolescent pour reconnaître «la» réalité et «sa» réalité. Par développement de l'autonomie, nous qualifions sa démarche personnelle pour prendre conscience de ses capacités, reprendre confiance en lui-même et transformer son comportement.

Si cette approche est appliquée à tous, «sur le plancher» elle prend évidemment des formes très différentes, les problèmes, le potentiel et la volonté de changement étant uniques à chaque adolescent. C'est pourquoi la structure clinique - les programmes, les activités, l'intervention des éducateurs, le «tempo» adopté - est adaptée à l'individu.

Cette approche individualisée commence dès avant l'admission. Non seulement le jeune est-il rencontré personnellement, mais on lui demande de remplir un questionnaire qui lui permet de se présenter et de nous donner son point de vue sur différentes questions.

Cette présentation est soumise au Comité d'admission en même temps que la «Synthèse du dossier», préparée par l'éducateur qui a rencontré le jeune, avec la participation des autres intervenants (travailleur social, éducateur du centre précédent, psychologue, orienteur).

Au moment où il est admis à Cité, le jeune n'est donc pas un dossier avec un numéro, mais un individu qui a des difficultés et des attentes bien définies et identifiées.

Cette connaissance préliminaire du jeune permet déjà de l'insérer dans une unité de vie, un programme scolaire ou professionnel, des activités de loisir qui correspondent à ses besoins et à ses attentes. 
Tout au long de son séjour, cette même approche «taillée sur mesure» se poursuivra et s'intensifiera. Ainsi, dès son arrivée, il pourra avoir un éducateur-parrain, qui développera avec lui une relation privilégiée et aura pour tâche autant de véhiculer et d'expliquer au jeune ce que les autres éducateurs attendent de lui que d'exposer à ces derniers les besoins et les attentes de l'adolescent.

Même si les huit éducateurs qui travaillent quotidiennement avec les douze adolescents de l'unité arrivent très vite à avoir une connaissance intime de chacun, la présence de ce parrain est en quelque sorte une garantie supplémentaire qu'une attention constante est portée à chaque adolescent. Ainsi, c'est son parrain qui aidera le jeune à se préparer à cette étape cruciale du comité multidisciplinaire. C'est également lui qui recueillera les observations quotidiennes faites par les autres éducateurs de l'unité, en fera l'analyse lors des réunions d'équipe et sera chargé de rappeler à tous les objectifs particuliers et ponctuels de l'intervention auprès de cet adolescent.

Ce parrainage est soutenu par des instruments cliniques, au premier rang desquels figure le «cahier clinique», qui permet à chaque éducateur, en tout temps, d'avoir à portée de la main non seulement une «histoire» de l'adolescent, mais aussi une analyse de son évolution récente et un rappel des objectifs précis sur lesquels celui-ci a accepté de travailler à court terme.

\section{AUTONOMIE ET RESPONSABILITÉ}

Faut-il le rappeler, l'un des buts du processus de réadaptation est d'amener l'adolescent à «s'assumer». Il y parviendra en retrouvant d'abord l'estime de soi et la confiance dans ses propres capacités, puis en apprenant à être moins dépendant du jugement des autres. En ce sens, la structure clinique de Cité des Prairies, même si elle doit tenir compte des impératifs d'encadrement, est donc conçue pour laisser place à l'initiative et au développement des responsabilités personnelles.

Cet apprentissage de l'autonomie, aussi fondamental soit-il, se fera cependant à travers un ensemble de gains et d'étapes souvent modestes mais toujours très concrets. À Cité des Prairies, l'autonomie est quelque chose qui s'acquiert, qui se gagne, au même rythme que se développe le sens des responsabilités. Plus le jeune se montrera responsable, face à lui-même et face aux autres, plus il verra s'agrandir les champs où peut s'exercer son entière liberté. 
Comme nous l'avons mentionné, ce double apprentissage se réalise à travers une «foule de petites choses», qui vont de la décoration des murs de la chambre à la possibilité de circuler de plus en plus librement dans le Centre et d'avoir accès à des activités de moins en moins encadrées. À mesure que le jeune exercera ses responsabilités, tant au niveau des programmes et des activités dont il fait partie qu'au sein de son unité de vie; il verra ses privilèges personnels et sa liberté de choix s'accroître. Cela commencera par la possibilité de pratiquer une activité ou un sport individuel, pour déboucher sur une sortie en petit groupe. Puis, alors que cela n'était pas prévu, il pourra se voir accorder un congé provisoire dans sa famille.

Ces mesures, qui visent à stimuler la responsabilité et l'autonomie individuelles, franchissent présentement une autre étape avec l'entrée en vigueur d'une nouvelle politique sur la «rémunération» des adolescents.

On avait déjà commencé, 11 y a quelques années, à inciter l'adolescent à ne pas compter uniquement sur la modeste «prime» que lui versait le Centre pour défrayer «ses besoins personnels». Ainsi, le jeune pouvait arrondir son "pécule» en faisant certaines tâches rémunérées. À compter de septembre 87 , ce principe sera développé.

D'une part, les produits de consommation «de luxe» (friandises, cigarettes, etc.), faisant jusque-là l'objet de «subsides» importants de la part du Centre, seront ramenés à des prix plus proches de la réalité, ce qui en réduira évidemment l'accès à l'adolescent qui aura choisi de vivre» sur sa seule «prime». Mais, d'autre part, l'adolescent qui le désire pourra compter sur un éventail beaucoup plus large de tâches rémunérées, qui lui donneront la possibilité de combler davantage de «besoins personnels» que ne le permettra dorénavant la prime miniminale.

On l'aura compris, il ne s'agit pas ici de «limiter la qualité de vie» des jeunes, il s'agit de leur faire prendre conscience de leur part de responsabilité individuelle face à la réalité qu'ils retrouveront à l'extérieur, où, là aussi, ils auront parfois le choix d'attendre passivement la «prime mensuelle minimale» ou d'améliorer volontairement leur sort.

Toute cette démarche vers la responsabilité et l'autonomie individuelles peut paraître naïve ou paternaliste. Il faut cependant se rappeler ce que signifie concrètement le mandat d'offrir des services de garde fermée et l'obligation de respecter une ordonnance du tribunal pour 
apprécier l'effort considérable que de telles mesures exigent des éducateurs: il est tellement plus simple d'imposer des règlements, de fournir un «encadrement général» et de servir à tous la même médecine que de chercher, dans le respect des particularités, à inciter chacun à trouver sa propre voie vers l'autonomie.

\section{UN CODE D'ÉTHIQUE}

Même si le respect des adolescents - respect de leurs problèmes, de leur potentiel, de leur volonté - est à la base même de la formation qu'ont reçue les éducateurs qui travaillent au Centre, Cité des Prairies a tenu à souligner l'importance qu'elle y accorde en proposant à tous ses employés - éducateurs et personnel d'encadrement et de soutien - un code d'éthique dont plusieurs points traitent spécifiquement de l'esprit qui doit les animer dans leurs relations avec les bénéficiaires.

On y traite spécifiquement du maintien «d'un standard de conduite personnelle qui respecte l'intégrité physique et morale des bénéficiaires». On y rappelle qu' «en tout temps, l'employé doit être conscient du modèle d'identification qu'il constitue pour le bénéficiaire».

Le code d'éthique insiste sur la confidentialité qui doit marquer les rapports de l'employé et des bénéficiaires. Il traite enfin de façon exhaustive des mesures disciplinaires qui «doivent être les moins contraignantes possible» et «proportionnelles au degré de gravité de l'infraction", interdisant même explicitement certaines de ces mesures.

\section{L'IMPLICATION DU COMITÉ DES BÉNÉFICIAIRES}

\section{LA STRUCTURE CIVIQUE}

Nous avons vu que l'engagement de l'adolescent dans son plan d'intervention est une condition sine qua non à la réussite de cette intervention. Dans le même esprit, on peut dire que la participation des jeunes à la vie de leur unité et à la marche générale du Centre est devenue un rouage essentiel à la réalisation du mandat de réadaptation de l'établissement. À Cité des Prairies, cet engagement des jeunes se situe dans une perspective de responsabilisation et de développement des compétences et, depuis quelques années, il a été structuré tant au niveau de ses objectifs que de son fonctionnement. C'est ce que l'on appelle la structure civique. 


\section{AU NIVEAU DE L'UNITÉ DE VIE}

La contribution des adolescents se situe d'abord au niveau de leurs préoccupations les plus immédiates. Dans chaque unité, le secteur vital s'occupe de tout ce qui regarde l'alimentation - le petit déjeuner et les collations sont pris au sein de chaque unité, - la cantine, le ménage, la buanderie, l'entretien et les réparations. C'est ce secteur qui participe à la gestion du budget annuel de $6800 \$$ confié à chaque unité pour les domaines énumérés ci-haut. C'est à la fois le ministère des Approvisionnements et le Conseil du trésor de l'unité.

Le secteur de vie de groupe s'intéresse pour sa part à l'animation des activités au sein de l'unité. C'est lui qui organise les sorties, prépare des événements spéciaux et, de façon générale, veille à développer un esprit de groupe au sein de l'unité.

Ces comités de secteurs sont formés des jeunes qui désirent y adhérer et sont responsables devant l'assemblée générale, formée de tous les jeunes de l'unité, à qui ils rendent des comptes hebdomadairement lors de la réunion civique. Les fonctions de responsabilité sont électives et renouvelables tous les trois mois.

\section{AU NIVEAU DE L'ÉTABLISSEMENT}

Au niveau de l'établissement, le rôle de coordination appartient à un comité qui regroupe les présidents de chaque unité. À ce rôle de coordination se greffe une fonction de consultation à l'orientation générale du Centre.

À plusieurs occasions, récemment, le Conseil d'administration et la direction générale de Cité des Prairies ont effectué une consultation générale des jeunes à travers cette structure civique. Sur différents dossiers - les visites, les sorties, le fumage - le processus de consultation a été le suivant:

Le directeur général a rencontré les présidents d'unité pour leur faire part de l'objet de la consultation, leur expliquer l'ancienne politique, les contraintes légales ou réglementaires dont l'établissement devait tenir compte, et les grandes lignes du projet de la direction.

Puis s'est enclenchée la procédure de consultation proprement dite, souvent à l'aide d'un sondage écrit. Après compilation des résultats et discussion des propositions dans chaque unité, le comité est revenu présenter - et défendre - ses recommandations au directeur général. 
Cela a réservé quelques surprises à la direction. Ainsi, contrairement à ce qu'on avait imaginé, la consultation sur les visites a suscité peu d'intérêt, les jeunes se disant dans l'ensemble satisfaits des règlements existants. En revanche, la politique régissant les sorties a soulevé plus de débats. De façon générale, le Conseil d'administration a tenu compte des opinions des jeunes, tout en tentant de les rendre compatibles avec les résultats des consultations parallèles menées auprès des parents et du personnel. Un exemple: alors que le projet de la direction concernant le fumage dans l'établissement n'avait pas osé proposer l'interdiction de fumer dans la cafétéria, de peur de heurter la volonté des adolescents, ce sont eux qui l'ont demandée et obtenue, malgré le désir contraire exprimé par le personnel, qui s'est finalement rallié à cette position.

Un dernier exemple, pour montrer que cette structure est loin d'être de tout repos pour la direction.

Au printemps 87, le comité de vie de groupe a participé à l'organisation d'une joumée «portes ouvertes» (enfin, pas toutes), pendant laquelle toutes les unités se sont mutuellement rendues visite, histoire de comparer les installations et les règles de vie. Cette journée s'est terminée par une assemblée générale où la direction s'est retrouvée sur la sellette lorsque les jeunes lui ont souligné quelques «contradictions» d'une unité à l'autre concernant l'application du règlement sur l'affichage dans les chambres, sujet qui leur tient particulièrement à cœur. En termes polis, mais non moins clairs, les autorités ont alors été priées de faire, à leur tour, preuve de cohérence.

Avec les années, cette structure civique prend de plus en plus d'ampleur et est devenue un outil privilégié à la fois pour faire prendre conscience au jeune de ses compétences - lui aussi a son mot à dire et ses idées à défendre - et pour lui permettre d'expérimenter différents niveaux de responsabilités.

\section{INTÉGRATION ET RÉINTÉGRATION SOCIALE}

Nous avons eu l'occasion, aux sections 1 et 2 , de décrire le système mis en place pour assurer au bénéficiaire des services intégrés, qui répondent à ses besoins particuliers à chaque phase de l'intervention. Un des objectifs de la structure clinique de Cité des Prairies est précisément de favoriser «un plan d'intervention axé sur un continuum de services et une utilisation de la mesure appropriée à toutes les phases de l'intervention». 
Nous ne voulons pas répéter ici tout ce qui a déjà été mentionné dans les sections précédentes relativement à l'intégration des services. Nous croyons, d'autre part, que cette intégration ressort clairement de la structure clinique elle-même. Nous aimerions donc nous attarder, dans la présente section, à décrire les actions posées en vue de favoriser la réintégration sociale des adolescents dont nous avons la garde.

\section{DE L'INSTITUTION À LA COMMUNAUTÉ}

Depuis bientôt sept ans, la réintégration sociale des jeunes pris en charge par Cité des Prairies a été définie clairement comme l'une des priorités de l'établissement. À tel point qu'on entend parfois dire que notre Centre, théoriquement l'un des plus «fermés» du réseau, en est devenu, à certains points de vue, l'un des plus ouverts.

Cet accent mis sur la réintégration des jeunes dans la société s'est en quelque sorte imposé de lui-même quand on a constaté que tous les efforts de réadaptation de l'adolescent accomplis «à l'interne», dans un milieu hautement structuré et "surprotégé», risquaient d'être annihilés lorsque le jeune quittait l'institution et se retrouvait dans le «vrai monde».

Avec la participation active des parents, concernés au premier chef par ce danger, Cité des Prairies a réorienté son action en adoptant trois types de mesures visant à préparer le jeune à affronter cette réalité et à lui assurer une transition en douceur.

\section{UNE FORMATION QUI COLLE À LA RÉALITÉ}

Nos efforts ont d'abord porté sur le processus de réadaptation luimême. Si le but premier des programmes et des activités est d'aider le jeune à se responsabiliser et à se prendre en main, il n'y a rien qui s'oppose à ce que ces buts soient atteints à travers des programmes qui auraient également pour conséquences de mieux l'outiller pour se tailler une meilleure place dans la société. Pour prendre un exemple précis, si des activités de céramique peuvent avoir un effet thérapeutique, pourquoi des activités plus proches de la réalité du travail n'auraient-elles pas un effet similaire?

En se basant sur ce principe, Cité des Prairies a donc développé deux types de programmes de formation, axés sur la réalité vécue par les adolescents: l'école et le travail.

La formation académique, offerte par la CECM selon le système des fiches individualisées, permet à un nombre appréciable de jeunes 
de rattraper souvent en 12 ou 18 mois les deux ou trois années de retard académique qu'ils avaient accumulées. À leur sortie, ceux qui en ont les capacités et le désir peuvent donc réintégrer immédiatement le réseau scolaire régulier ou celui des écoles pour décrocheurs, à un niveau normal pour leur âge.

Plusieurs de nos jeunes, même s'ils ont effectué un certain rattrapage académique au Centre, préfêrent cependant gagner immédiatement le marché du travail. Dans le contexte économique actuel, c'est une décision qui se heurtera à des difficultés majeures, qui peuvent évidemment influer fortement sur la résolution du jeune de s'en sortir par le travail plutôt que par la délinquance.

Cité des Prairies a choisi d'affronter cette réalité «réalistement». La formation professionnelle que nous donnons aux adolescents tiendra donc compte de leurs capacités et du genre de travail auquel ils peuvent aspirer à leur arrivée sur le marché de l'emploi. C'est dans cet esprit que des programmes de soudure, de menuiserie, d'hôtellerie, d'électrotechnique et de pâtisserie-boulangerie ont été développés.

Ces programmes de formation professionnelle poursuivent essentiellement deux objectifs: l'apprentissage d'une technique, bien sûr, mais aussi - on pourrait dire surtout - l'acquisition d'habitudes de travail, qui font peut-être davantage défaut à nos adolescents que l'expertise.

L'expérience nous a démontré que la principale difficulté rencontrée par le jeune, à sa sortie du Centre, n'était pas tant de trouver un job que de le garder. En effet, les échecs que connaissent ces nouveaux venus sur le marché du travail ne concernent pas d'abord leur compétence mais le manque de discipline dont ils font parfois preuve au niveau de la ponctualité ou des relations avec l'autorité.

Sous la supervision simultanée d'un moniteur, qui leur apprend leur métier, et d'un éducateur, qui leur apprend ce qu'il faut faire pour le conserver, les jeunes qui ont choisi la formation professionnelle pourront donc quitter Cité des Prairies un peu mieux outillés pour affronter la vie «normale» qui les attend.

\section{PRÉPARER LA «SORTIE»}

Cette importance accordée à la réinsertion sociale a évidemment provoqué des changements au niveau même des programmes de réadaptation. S'il demeurait essentiel de travailler intensément avec le 
jeune sur ses difficultés personnelles pendant qu'il était à Cité, ce travail devait maintenant se faire avec un but plus lointain, en pensant au jeune lorsqu'il n'y sera plus.

Concrètement, cela signifie donc, dès les premiers mois du séjour, un ensemble de mesures qui préparent déjà l'adolescent à quitter l'institution. Dans cet esprit, le dosage gradué des sorties, des congés et des séjours dans la famille peut devenir un test important pour mesurer le degré de préparation du jeune à rejoindre la communauté.

Le choix de l'unité de vie s'en trouve également influencé. Ainsi, il existe présentement un programme regroupant 16 adolescents, axé spécifiquement vers une préparation à l'externat.

\section{L'INTERVENTION «HORS LES MURS»}

Malgré ces programmes de formation et cette préparation à la «transition», qui se déroulent à l'intérieur même du Centre, on a vite réalisé que le cadre protecteur de l'établissement devait être dépassé et que la véritable transition devait être expérimentée dans un contexte plus ouvert, plus réaliste. Ce faisant, on a toutefois voulu éviter que cette première «brisure» entre «interne» et «externe» soit accentuée par la deuxième brisure que constitue souvent l'arrivée auprès du jeune d'une deuxième équipe d'intervenants, rattachés à un autre centre.

C'est pour ces motifs que Cité des Prairies, d'abord connue pour son mandat de «garde fermée», a été appelée à poursuivre son action à l'extérieur et à étendre ses services. Pour répondre aux besoins divers de nos adolescents toutefois, cette intervention externe, tout en faisant partie d'un continuum de services, s'est fixée différents objectifs et a emprunté un éventail de moyens.

Ainsi, certains jeunes, même s'ils ont réintégré leur famille, peuvent poursuivre leurs études à Cité, dans le cadre du Centre de jour qui accueille douze d'entre eux.

D'autres, qui ont choisi le travail, peuvent trouver un premier emploi et acquérir ainsi de l'expérience dans deux ateliers de production - en menuiserie et en rembourrage -. où ils doivent fournir un effort identique à celui qui sera exigé d'eux dans toute entreprise privée.

Le passage du jeune «de la serre à la jungle» ne signifie pas qu'il cesse d'avoir besoin du soutien et de l'encadrement des éducateurs. Qu'il ait choisi de poursuivre ses études ou de gagner le marché du 
travail, l'adolescent pourra donc compter sur un programme d'intervention - Les sentiers - qui a pour but de répondre aux besoins particuliers de chacun et de renforcer ses habitudes de travail et de vie. Cela pourra toucher l'assiduité et la ponctualité au travail aussi bien que l'occupation des temps libres, moments difficiles pendant lesquels l'adolescent qui a choisi de commencer une nouvelle vie hors de son ancien milieu se retrouve isolé et particulièrent vulnérable.

L'action du Centre auprès de ceux qui viennent de quitter ses murs ne s'arrête pas au soutien de l'acquisition de «nouvelles habitudes». Le problème de l'hébergement étant une dimension importante du quotidien de ceux qui ne vivent pas au sein de leur famille, Cité des Prairies a également choisi d'intervenir à ce niveau. Il existe présentement deux foyers de groupe, pour ces adolescents qui préfèrent ne pas vivre dans leur famille mais qui ne se sentent pas encore prêts à vivre seul en appartement. Un foyer similaire existe aussi pour les jeunes adultes, auprès de qui Cité des Prairies n'a plus de responsabilité légale, mais qui demandent d'eux-mêmes notre support pour mieux asseoir leurs efforts de réinsertion.

Enfin, Cité des Prairies peut également assurer l'hébergement, l'encadrement et le suivi intensif à des jeunes qui viennent de quitter le centre d'accueil mais qui font face à des difficultés importantes autant au niveau de leur motivation au travail que l'organisation de leur vie quotidienne. «La Relève», située au cœur de Rosemont, vient donc en aide à ces jeunes que des difficultés particulières d'adaptation rendent éminemment vulnérables à une «rechute».

S'il fallait caractériser en un mot la politique qui guide l'action de Cité des Prairies au niveau de la réintégration sociale des adolescents, c'est donc celui de «continuité» qui s'imposerait. Continuité entre réadaptation et réinsertion, bien sûr, mais aussi continuité dans l'intervention, le jeune étant maintenant accompagné dans cette phase délicate de la réinsertion sociale par des intervenants qui connaissent bien ses forces et ses besoins.

\section{CONCLUSION}

La Cité des Prairies fête cette année ses 25 ans d'existence.

Ceux qui ont connu l'ère «Berthelet» seront en mesure de mesurer le progrès accompli par cet établissement pour offrir à des jeunes que plusieurs considèrent encore comme «irrécupérables» un programme de réadaptation structuré, qui constitue souvent leur dernière chance. 
Les efforts réalisés depuis maintenant plus de 12 ans pour humaniser cette «prison pour adolescents» et en faire un véritable centre de réadaptation et de réinsertion sociale ont toujours reposé sur un principe relativement simple: le respect.

Respect des difficultés qu'il affronte, respect de ses blessures profondes, respect de ses lacunes et de ses résistances. Respect, aussi, de ses espoirs et de ses capacités de s'en sortir. Respect, surtout, du rythme et de la démarche qu'il choisira pour se libérer.

Ces efforts pour humaniser cette «prison pour adolescents» et en faire un véritable centre de réadaptation et de réinsertion sociale reposent également sur la qualité et l'engagement profond des intervenants qui jour après jour continuent, malgré les nombreuses difficultés qu'ils rencontrent, d'avoir foi dans les possibilités d'évolution de cette clientèle de «bout de ligne» 